Javier Federico García Meana

Enfermero especialista en Geriatría y Gerontología. Hospital Virgen del Valle. Toledo. España.

Correo electrónico: fede88624@hotmail.com

Recibido el 25 de febrero de 2019; aceptado el 21 de junio de 2019

\section{Utilización del sevoflurano de forma tópica en úlceras de la extremidad inferior: revisión sistematizada}

\section{RESUMEN}

Las úlceras de la extremidad inferior siguen siendo en la actualidad un problema global. Las opciones analgésicas para el control del dolor se basan generalmente en medidas farmacológicas con acción local y/o sistémica. El sevoflurano es un anestésico general inhalatorio, asociado a sus efectos sobre el sistema nervioso central, y tradicionalmente no se emplea por otras vías que no sea inhalado. Sin embargo, hoy en día se le conoce una acción analgésica a nivel central y también periférico. Actualmente, su uso clínico ha llevado a algunos autores a considerar la posibilidad de nuevos efectos del sevoflurano a través de la vía tópica. Objetivo: Sintetizar las evidencias científicas disponibles sobre el uso del sevoflurano aplicado de forma tópica en úlceras de la extremidad inferior. Metodología: Revisión sistematizada de la literatura científica, siguiendo la guía PRISMA. La búsqueda de estudios se realizó en las principales bases de datos bibliográficas, sin límite de fechas ni de idiomas. También se realizó una búsqueda incluyendo resúmenes de congresos. Resultados: Se obtuvieron un total de 120 referencias. Finalmente, ocho de ellas correspondían a los estudios incluidos para la síntesis cualitativa. En la mayoría de los estudios se encontró una disminución del dolor de 8 a 2 puntos en las escalas empleadas. Conclusiones: Los escasos estudios parecen sugerir un importante efecto analgésico aplicado de forma tópica, un probable efecto antibacteriano y un posible efecto promotor de la cicatrización. Sin embargo, son necesarios más estudios comparativos con un tamaño de muestra mayor, con mejor calidad en sus diseños.

PALABRAS CLAVE: Úlcera de la pierna, sevoflurano, revisión sistematizada.

\section{ABSTRACT}

Leg ulcers are a global problem daily. The analgesic options for pain control are generally based on pharmacological measures with local and / or systemic action. Sevoflurane is a general inhalation anesthetic, associated with its effects on the central nervous system, its use not being traditional by other routes that are not inhaled. However, today it is known an analgesic action at the central level and at the peripheral level. Actually, the clinical use of this product has led some authors to consider the possibility of new effects of Sevoflurane topically. Objective: To synthesize the available scientific evidences about the use of Sevoflurane topically on leg ulcers. Methodology: Systematized review of the scientific literature, following the PRISMA guide. The main bibliographic databases were searched without date or language limits. Also references lists and congress abstracts were searched. Results: 120 references were identified. Finally, 8 of them were selected for qualitative synthesis. In most studies, was found a decrease in pain of 8 to 2 points in the scales used. Conclusions: The few studies suggest an analgesic effect applied topically, a probable antibacterial effect and a possible healing promoting effect. However, comparative studies of large sample are needed, with a better quality designs.

KEYWORDS: Leg ulcer, Sevoflurane, systematized review.

\section{У INTRODUCCIÓN}

Las úlceras de la extremidad inferior (UEEII) han sido un problema de salud a lo largo de la historia y siguen siendo, en la actualidad, un problema global. Afectan al 1-3\% de la población, con altos costes involucrados en su tratamiento; constituyen aproximadamente el 2,5\% de los presupuestos totales de la atención sanitaria en Europa y América ${ }^{1}$.

Según el Primer Estudio Nacional de Prevalencia de Úlceras de Pierna en Espańa, realizado por el GNEAUPP en el ańo 2002, la prevalencia de UEEII en mayores de 14 años fue del 0,156\%. Un 0,09\% correspondió a úlceras de etiología venosa; un 0,013\%, a úlceras de etiología arterial; un $0,026 \%$, a úlceras de etiología mixta, y un 0,027\% a úlceras de pie diabético. La prevalencia de estas úlceras de pie diabético en la población diabética se estimó en un $0,53 \%$. Esta prevalencia se triplica en la población mayor de 65 años en comparación con la población adulta ${ }^{2}$.

Dentro de las UEEII cabe destacar las úlceras de etiología venosa, ya que representan aproximadamente el $70 \%$ de todas las UEEII ${ }^{1}$. Son más frecuentes en mujeres $(67,2 \%)$ que en hombres $(32,8 \%)^{2}$, con una tasa de recurrencia que oscila en torno al $17 \%$ durante el primer ańo y entre el $55 \%$ y el $60 \%$ durante los primeros 5 ańos $^{3}$, por lo que constituyen un importante problema de salud por su frecuencia, alta tasa de recurrencia, carácter crónico y dolor. 
Este dolor de las UEEII es uno de los motivos que hace que su carácter permanente cause una limitación funcional y aleje poco a poco al paciente del desempeño de las actividades básicas e instrumentales de la vida diaria. Puede producir efectos negativos física, psíquica y socialmente. En definitiva: influye directamente en la calidad de vida ${ }^{4,5}$. Las opciones analgésicas para el control del dolor asociado a estas úlceras se basan generalmente en medidas farmacológicas. Entre ellas se incluyen:

- La administración de anestésicos locales ${ }^{6}$ por vía tópica (mepivacaína al 3,5\%)

- La administración de diversos fármacos con acción analgésica sistémica. Para el dolor moderado y ocasional: analgésicos no opioides; y para el dolor intenso y constante: opiáceos y derivados en sus diferentes formas de presentación, contribuyendo directamente al control analgésico global, aunque pueden producir efectos no deseables ${ }^{7}$.

El sevoflurano es un anestésico general inhalatorio del grupo de los éteres halogenados, con un adecuado perfil de seguridad, indicado en la inducción y mantenimiento de la anestesia general en el proceso quirúrgico en adultos y niños. Dicho efecto se ha asociado a sus efectos sobre el sistema nervioso central, no siendo tradicional su empleo por otras vías que no sean inhaladas, al considerarse que no posee más efectos ${ }^{8}$.

Sin embargo, hoy en día se le conoce una acción analgésica a nivel central $^{9}$ y periférico ${ }^{10}$, aunque tradicionalmente se ha considerado que los anestésicos halogenados carecen de acción a este segundo nivel ${ }^{11}$.

Actualmente, el uso clínico de este producto ha llevado a algunos autores a considerar la posibilidad de nuevos efectos del sevoflurano a través de otras vías de administración. Más exactamente por vía tópica.

A la gran mayoría de los profesionales, la aplicación tópica de sevoflurano líquido (fuera de ficha técnica) sobre las UEEII para conseguir la analgesia puede resultar novedosa, cuando no sorprendente, por el simple hecho de que llevan más de 20 ańos administrando sevoflurano por vía inhalatoria y, hasta ahora, apenas se han cuestionado otras vías de administración ${ }^{10}$. Por ello, se plantea la siguiente pregunta: ¿proporciona nuevos efectos la aplicación del sevoflurano de forma tópica en UEEII?

\section{У OBJETIVO}

Sintetizar las evidencias científicas disponibles sobre el uso del sevoflurano aplicado de forma tópica en UEEII.

\section{Y METODOLOGÍA}

Revisión sistematizada de la literatura científica, siguiendo la Guía PRISMA.

Los apartados descritos en este documento se basan en la Declaración PRISMA $^{12}$

Aquellos que no aparecen reflejados no procedían en el estudio (http://www.prisma-statement.org/PRISMAStatement/Default.aspx).

\section{Protocolo y registro}

No existe un protocolo de revisión previo sobre este tema.

\section{Fuentes de información}

Se realizó una búsqueda bibliográfica desde el comienzo de su indexación hasta junio de 2018 en las principales bases de datos nacionales e internacionales.
Nacionales: CUIDEN Plus y ENFISPO.

Internacionales: Cochrane Library Plus, Medline (a través de PubMed), CINAHL, EMBASE, LILACS.

\section{Búsqueda}

Para la búsqueda en Medline, CINAHL y EMBASE se utilizaron los descriptores del Medical Subject Headings (MeSH), y para las demás bases, los descriptores propios de sus tesauros.

Se identificaron los estudios primarios relevantes, utilizando para ello un lenguaje controlado (tabla 1).

Tabla 1. Estrategia de búsqueda según las bases de datos

\begin{tabular}{|l|l|}
\hline & 1. Sevoflurane \\
\hline 2. Leg Ulcer \\
\hline 3. Ulcer \\
\hline Cochategia de húsqueda para & 4. Ulcers \\
\hline y EMBASE: & 5.1 AND 2 \\
\hline 6. 1 AND 3 \\
\hline 7. 1 AND 4 \\
\hline 1. Sevoflurano \\
\hline 2.Úlcera de la pierna \\
\hline Estrategia de búsqueda para & 3. Úlcera \\
\hline CUIDEN Plus, ENFISP0, LILACS: & 4. 1 AND 2 \\
\hline & 5.1 AND 3 \\
\hline
\end{tabular}

\section{SELECCIÓN DE LOS ESTUDIOS}

Todos aquellos estudios donde se utilizara el sevoflurano aplicado de forma tópica en las UEEII, sin restricción de idiomas. Los estudios fueron seleccionados a partir del título ${ }^{12}$ y del resumen y se obtuvieron a texto completo para un análisis más detenido.

\section{Proceso de extracción de datos}

La selección de referencias, inclusión de artículos y extracción de los datos ha sido realizado por la misma persona.

\section{Características de los estudios}

De cada estudio incluido se extrajeron: autores, año de publicación, país, participantes, intervención, comparación, resultados, diseńo del estudio, lugar de seguimiento, periodo de seguimiento y cantidad de sevoflurano utilizada.

\section{Síntesis de los datos}

Se ha realizado una síntesis utilizando estadística descriptiva de todos los estudios que incluían en sus resultados: nivel de dolor antes y después de la instilación de sevoflurano y duración de la analgesia tópica.

Para dichas variables se calcularon medidas de tendencia central (media y mediana) y medidas de dispersión (varianza y desviación estándar). Posteriormente, se realizó inferencia sobre la media, calculando 
el intervalo de confianza (IC) al 95\% con sus límites inferior y superior correspondientes.

\section{VESULTADOS}

\section{Selección de los estudios}

En total se encontraron 117 registros identificados mediante búsquedas en las diferentes bases de datos (fig. 1). El número de registros adicionales identificados mediante otras fuentes (congresos de la Sociedad Espańola de Heridas) fue $\mathrm{n}=3$.

El número de citas por duplicidad eliminadas fue 38 , siendo el número de registros cribados de 82, de los cuales se excluyeron 66 por no estar relacionado, en su resumen, con el objetivo a estudio.

El número de artículos a texto completo evaluados para su elegibilidad fue de 16, de los cuales se excluyeron 8:

- 4 por ser cartas al editor/director, como artículos de opinión, sin aportar nada.

- 4 por ser artículos con diferente título, pero mismo contenido que otro estudio ya incluido.

Finalmente, el número de estudios incluidos en la síntesis cualitativa fue de 8 .

En la tabla 2 se presentan las principales características de los 8 estudios seleccionados.

En los cuatro casos clínicos de la tabla 2 se estudió a participantes mayores de 60 años con UEEII de etiología venosa resistentes a analgesia local y/o sistémica. En todos ellos se aplicó sevoflurano de forma tópica (en can- tidades dispares). En dos de ellos, el seguimiento se realizó en ámbito hospitalario y en los otros, dos en domicilio. En cuanto a los resultados, en todos ellos se produjo una disminución del dolor (reflejado en la escala analgésica del dolor empleada). Únicamente se describió un único efecto adverso (irritación pruriginosa leve) en el caso clínico de Gerónimo-Pardo et al. ${ }^{13}$.

En la serie de casos de Imbernón et al. ${ }^{14}$ se incluyó a 3 participantes con UEEII infectadas por Pseudomonas aeruginosa y Staphylococcus aureus resistente a meticilina. Se instiló sevoflurano a una cantidad determinada de $1 \mathrm{ml} / \mathrm{cm}^{2}$ del tamaño de la úlcera. Este tratamiento se realizó cada 48 horas durante 30 días, sin utilización de ningún antibiótico por vía sistémica. A los 30 días se obtuvo la erradicación de la infección por $S$. aureus resistente a meticilina (cultivo negativo a los 30 días); sin embargo, la infección por $P$. aeruginosa persistió (cultivo positivo a los 30 días).

En las dos series de casos restantes, se incluyó a diversos pacientes con UEEII que presentaban dolor por encima de 4 puntos en las escalas del dolor empleadas. Se aplicó sevoflurano a una cantidad determinada de $1 \mathrm{ml} / \mathrm{cm}^{2}$ del tamaño de la úlcera, y como resultado se incluyó una disminución importante del dolor durante más de 10 horas. Se describieron como efectos adversos: irritación pruriginosa leve y eritema perilesional, entre otros.

Por último, en el estudio observacional prospectivo realizado por Dámaso Fernández-Ginés ${ }^{15}$ se incluyó a 15 pacientes con UEEII de etiología venosa, con dolor igual o superior a 7 puntos en la escala visual analógica (EVA). Estos 15 pacientes fueron asignados aleatoriamente en dos grupos en una proporción 2:1:

- Grupo $1(\mathrm{n}=10)$ : analgesia sistémica con morfina + limpieza con suero salino fisiológico + instilación líquida de sevoflurano $\left(1 \mathrm{ml} / \mathrm{cm}^{2}\right.$ del tamańo de la úlcera) + cuidado estándar.

- Grupo $2(\mathrm{n}=5)$ : analgesia sistémica con morfina + limpieza con suero fisiológico + cuidado estándar.

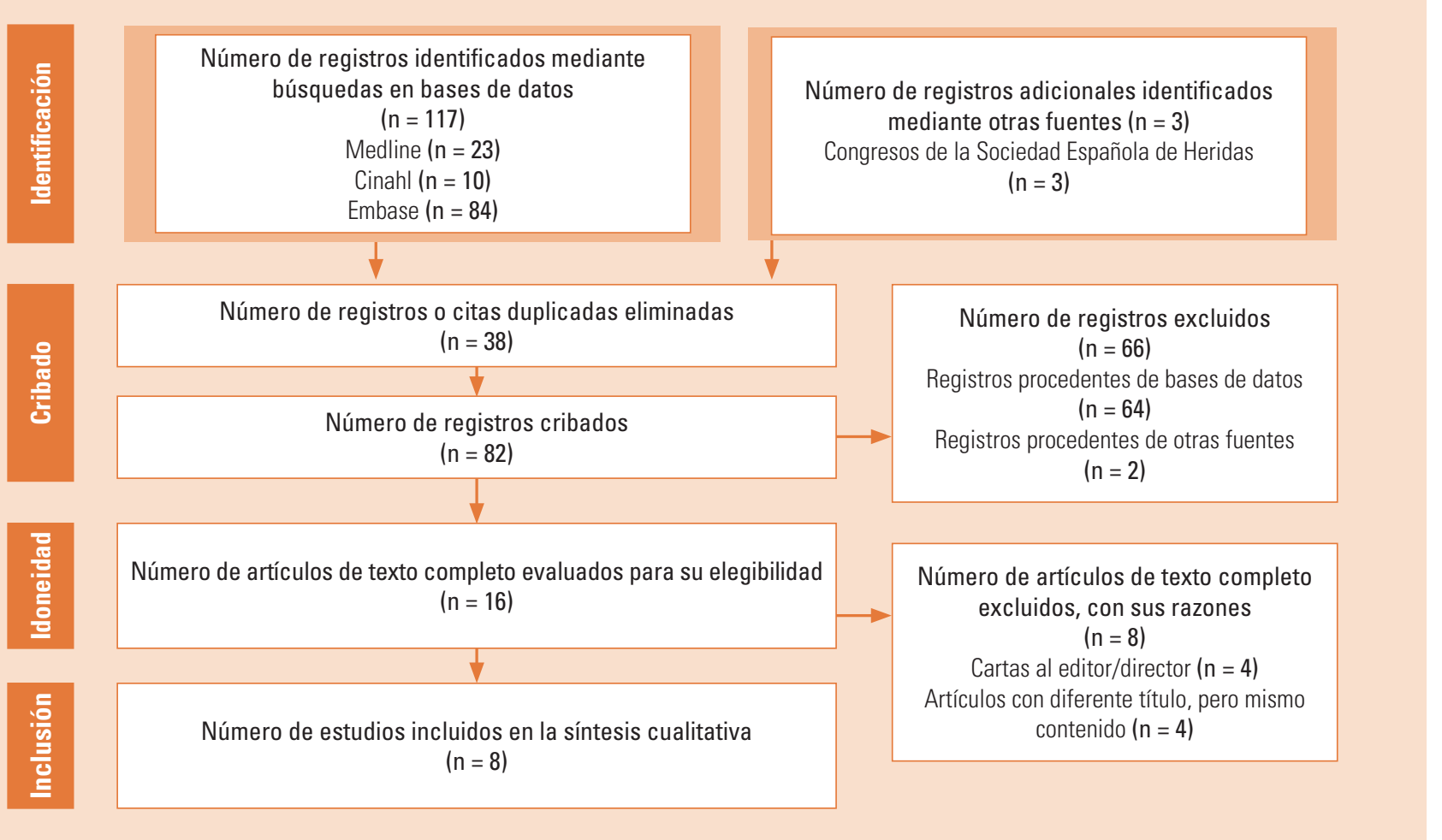

Figura 1. PRISMA 2009. Diagrama de flujo (versión española). 


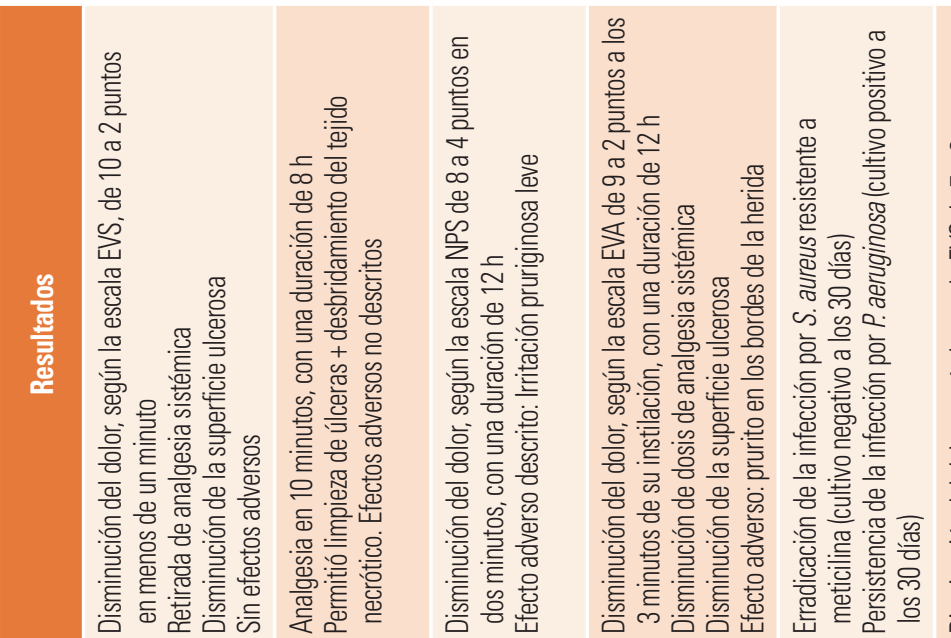

量 高鿷

高

H:

s

음

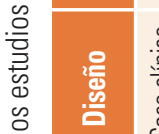

응

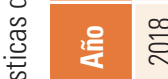

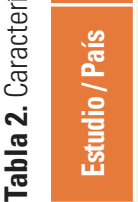

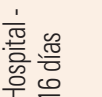

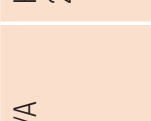

ta

$\stackrel{\frac{\pi}{2}}{\frac{s}{2}}$

六言

$\overline{\mathrm{E}}$

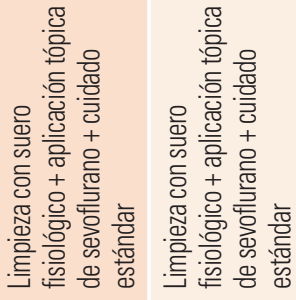

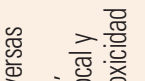

㩊

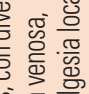

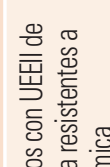

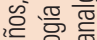

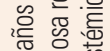

\%

)

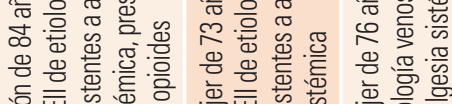

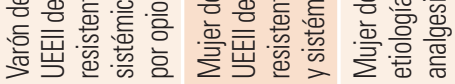

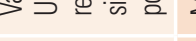
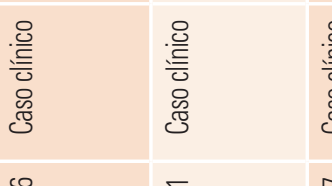

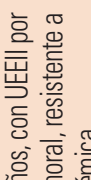

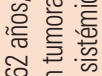

ฮิ

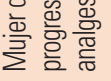

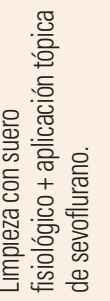

言

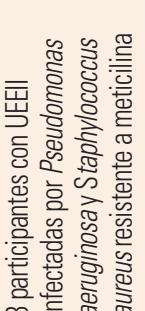

言高

兽

윻

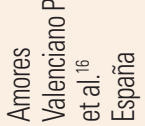

들. 동

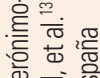

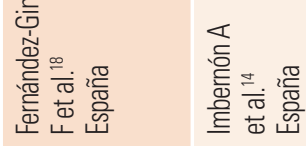

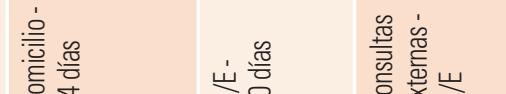

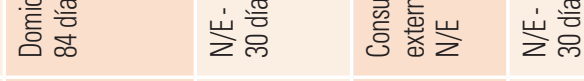

$\frac{\pi}{\frac{\pi}{2}} \frac{\frac{\pi}{2}}{\frac{\pi}{2}} \frac{\pi}{\frac{\pi}{2}}$

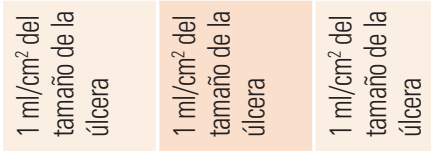

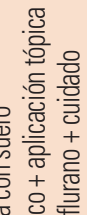

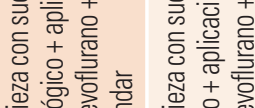

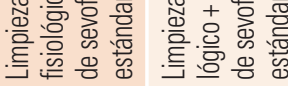

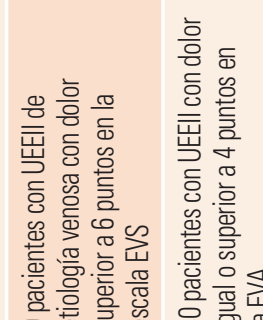

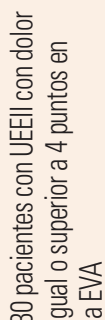

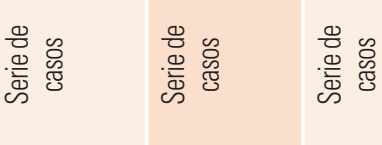

高 商 产

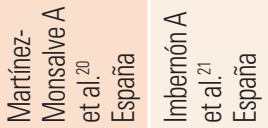

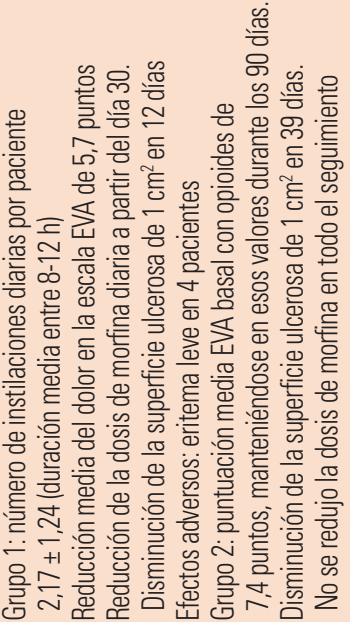

을

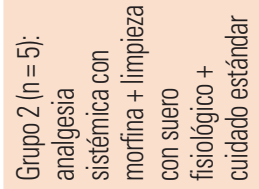

홍 $\frac{\pi}{8}$

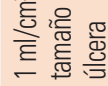

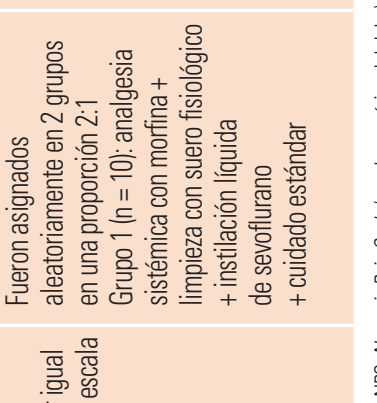

응 흥 $\frac{\pi}{ㄷ}$

岀 $\overline{0}$ 。

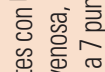

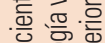

든은 을

产高唁

亏ิ

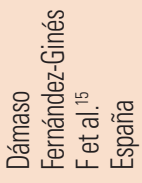


Se describieron los siguientes resultados:

Grupo 1: número de instilaciones diarias por paciente $2,17 \pm 1,24$ (duración media entre 8 y 12 h). Reducción del dolor en la EVA de 5,7 $\pm 2,0$ puntos $(p=0,001)$. Reducción de la dosis de morfina diaria a $20 \mathrm{mg} /$ día a partir del día 30. Se produjo una disminución de la superficie ulcerosa de $1 \mathrm{~cm}^{2}$ en 12,2 días. Efectos adversos: eritema leve en 4 pacientes.

Grupo 2: puntuación en la EVA basal con opioides de 7,41 \pm 1,49 puntos, valores que se mantuvieron durante todo el seguimiento. No se redujo la dosis de morfina en todo el seguimiento: $65 \mathrm{mg} / \mathrm{día}( \pm 5 \mathrm{mg})$. Se produjo una disminución de la superficie ulcerosa de $1 \mathrm{~cm}^{2}$ en 39,3 días.

El nivel del dolor medio según diferentes escalas que puntuaban entre 0 y 10 puntos, en todos los estudios que lo reflejaban $(n=5)$, fue:

- Antes de la instilación de sevoflurano: 8,5 puntos (IC 95\% 7,119,89).

- Después de la instilación de sevoflurano: 2,5 puntos (IC 95\% 1,42-3,57).

La duración media de la analgesia tópica del sevoflurano, en todos los estudios que lo reflejaban ( $\mathrm{n}=5$ ), fue de 10,94 horas (IC 95\% 8,7813,10).

\section{У DISCUSIÓN}

\section{Resumen de la evidencia}

El volumen de investigación sobre el sevoflurano aplicado de forma tópica es muy escaso. Únicamente se han encontrado casos clínicos aislados, series de casos y un estudio en el cual asignan dos grupos de forma aleatoria, pero no indican que sea un ensayo clínico, lo catalogan como observacional prospectivo (debe faltar algún trámite o alguna autorización que se desconoce, para poder catalogarlo como ensayo clínico).

Sería importante diseñar estudios con una metodología robusta para explorar las áreas de incertidumbre sobre el sevoflurano aplicado de forma tópica.

Con base en la investigación, se proponen tres áreas de posible utilidad clínica:

1. Efecto analgésico local.

2. Efecto antibacteriano.

3. Efecto promotor de la cicatrización.

\section{EFECTO ANALGÉSICO LOCAL}

Casi todos los estudios muestran que el sevoflurano aplicado de forma tópica disminuye el dolor (dolor resistente con analgesia sistémica) de forma rápida, intensa y duradera.

Podría tener un papel clave para el control del dolor, con apenas efectos secundarios, mejorando la efectividad real si se demuestra una mejor adherencia de los profesionales clínicos a su uso y una mejor tolerancia de los pacientes. Pero precisa de estudios más robustos.

\section{EFECTO ANTIBACTERIANO}

Un estudio muestra que el sevoflurano aplicado de forma tópica erradica la infección localizada causada por $S$. aureus resistente a meticilina a los 30 días, por lo que también podría tener un papel importante en este aspecto; pero se requieren de estudios más robustos en este aspecto que así lo demuestren.

\section{EFECTO PROMOTOR DE LA CICATRIZACIÓN}

En esta revisión, varios estudios muestran que el sevoflurano aplicado de forma tópica disminuye la superficie ulcerosa; pero esta disminución podría estar influenciada por el cuidado estándar aplicado posteriormente. Este aspecto requiere de más investigación.

No existe actualmente dosis recomendada para este tipo de uso; aunque la mayoría de los estudios muestran la cantidad de $1 \mathrm{ml} / \mathrm{cm}^{2}$, se siguen requiriendo más estudios para que así se recomiende.

No obstante, el sevoflurano presenta dos problemas ańadidos: en primer lugar, su elevado precio, que dificulta su uso habitual en la práctica; en segundo lugar, al tratarse de un anestésico inhalado y de fácil evaporación, se corre el riesgo de intoxicación o sufrir parte de sus efectos al usarlo.

\section{$\searrow$ LIMITACIONES}

La mayoría de los estudios son casos clínicos, series de casos y estudios observacionales.

\section{$\vee$ CONCLUSIONES}

Si bien los escasos estudios parecen sugerir un importante efecto analgésico aplicado de forma tópica ${ }^{16,17}$, un probable efecto antibacteriano ${ }^{14}$ y un posible efecto promotor de la cicatrización ${ }^{15,16,18}$, quedan por concretar muchos aspectos (dosis, pautas, márgenes terapéuticos, etc.) que permitan concretar su utilización idónea en esta forma de aplicación.

Puede ser una buena opción analgésica en UEEII dolorosas, e incluso existe la posibilidad de poder extrapolarse a otras úlceras dolorosas: mejorando el confort, infiriendo en la calidad de vida o en proporcionar una muerte digna; evitando con ello tratamientos sistémicos, invasivos y en definitiva a un sufrimiento diario ${ }^{19}$.

Serían necesarios más estudios comparativos con muestra de mayor tamańo, con mejor calidad en sus diseños, de forma que sea posible la realización de un metaanálisis a posteriori

\section{Financiación}

No existen fuentes de financiación para la realización de dicha revisión.

\section{Conflicto de intereses}

El autor declara no tener ningún conflicto de intereses.

\section{Agradecimientos}

A Raquel Sarabia Lavín por su disponibilidad, paciencia y conocimientos. A Belén Cámara Marín por su paciencia, apoyo y cariño.

\section{У BIBLIOGRAFÍA}

1. Parker CN, Finlayson KJ, Shuter P. Edwards HE Risk factors for delayed healing in venous leg ulcers: a review of the literature. Int J Clin Pract. 2015;69(9):967-77.
GNEAUPP-UICF-Smith \& Nephew 2002-2003. Epidemiología de las úlceras venosas, arteriales, mixtas y de pie diabético. Gerokomos. 2004;15(4):230-47. 
3. Gohel MS, Taylor M, Earnshaw JJ, Heather BP, Poskitt KR, Whyman MR. Risk factors for delayed healing and recurrence of chronic venous leg ulcers--an analysis of 1324 legs. Eur J Vasc Endovasc Surg. 2005:29(1):74-7.

4. Dantas Liberato SM, Gomes de Souza AJ, Fernandes-Costa IK, De Vasconcelos-Torres G, Fortes-Vitor A, Brandão de Carvalho-Lira AL. A enfermagem no manejo da dor em pessoas com úlcera venosa: revisão integrativa Nursing in the management of pain in people with venous ulcer: integrative review. Rev Pesqui Cuid é Fundam Online [Internet]. 2016 [citado 26 de febrero de 2018];8(2):4109-20. Disponible en: http://seer.unirio.br/index. php/cuidadofundamental/article/view/3251

5. Cardoso-Tavares AP, Chaves-Sá SP, Guitton-R. B. de Oliveira BG, Sousa AS. Quality of life of elderly patients with leg ulcers. Esc Anna Nery. 2017;21(4)

6. Marinel.Io Roura J VSJ, coords. Conferencia nacional de consenso sobre las úlceras de la extremidad inferior (C.O.N.U.E.I.).

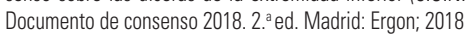

7. Traber J, Held U, Signer M, Huebner T, Arndt S, Neff TA. Analgesic efficacy of equimolar $50 \%$ nitrous oxide/oxygen gas premix (Kalinox®) as compared with a $5 \%$ eutectic mixture of lidocaine/ prilocaine (EMLAB) in chronic leg ulcer debridement. Int Wound J. 2017:14(4):606-15

8. Agencia Española de Medicamentos y Productos Sanitarios. Mi- nisterio de Sanidad, Política Social e Igualdad. Ficha técnica del Sevoflurano. 2016

9. Matute E, Rivera-Arconada I, López-García JA. Effects of propofol and sevoflurane on the excitability of rat spinal motoneurones and nociceptive reflexes in vitro. Br J Anaesth. 2004;93(3):422-7.

10. Gerónimo Pardo M, Martinez Serrano M. Usos alternativos del sevoflurano. Efecto analgésico tópico. Rev Electron Anest R. [Internet]. 2012 [citado 27 de junio de 2018];4(4):181. Disponible en: https://anestesiar.org/2012/usos-alternativos-del-sevoflurano-efecto-analgesico-topico/

11. Antognini JF, Kien ND. Potency (minimum alveolar anesthetic concentration) of isoflurane is independent of peripheral anesthetic effects. Anesth Analg. 1995;81(1):69-72.

12. García-Fernández FP, Pancorbo-Hidalgo P, Verdú-Soriano J. Efectividad de la Película Barrera No Irritante en la prevención de lesiones de piel. Revisión sistemática. Gerokomos. 2009;20(1):29-40.

13. Gerónimo-Pardo M, Martínez-Monsalve A, Martínez-Serrano M, Gerónimo-Pardo KM. Analgesic effect of topical sevoflurane on venous leg ulcer with intractable pain. Phlebologie. 2011;40:95-7.

14. Imbernón-Moya A, Ortiz-de Frutos FJ, Sanjuan-Alvarez M, Portero-Sanchez I, Merinero-Palomares R, Alcazar V. Topical sevoflurane for chronic venous ulcers infected by multi-drug-resistant organisms. Int Wound J. 2017:14(6):1388-90.

15. Dámaso Fernández-Ginés F, Cortiñas-Sáenz M, Mateo-Ca- rrasco $H$, de Aranda AN-G, Navarro-Muñoz E, Rodríguez-Carmona $R$, et al. Efficacy and safety of topical sevoflurane in the treatment of chronic skin ulcers. Am J Heal Pharm. 2017;74(9):e176-82

16. Amores Valenciano P, Navarro Carrillo A, Romero Cebrián MA Gerónimo-Pardo M. Topical sevoflurane for rescue analgesia in refractory pain due to chronic venous ulcers. Emergencias. 2018:30(2):138.

17. Imbernón A, Blázquez C, Puebla A, Churruca M, Lobato A, Martínez $M$, et al. Chronic venous ulcer treatment with topical sevoflurane. Int Wound J. 2016;13(5):1060-2.

18. Fernández-Ginés FD, Cortiñas Sáenz M, Fernández Sánchez C Morales-Molina JA. Sevoflurano tópico: una nueva opción terapéutica paliativa en las úlceras cutáneas. Med Paliativa. 2017;24(2):104-8

19. Lernevall LSD, Fogh K, Nielsen CB, Dreyer PS. Lived experiences of life with a leg ulcer -a life in hell.EWMA J. 2017;17(1):15-21.

20. Martínez-Monsalve A, Gerónimo-Pardo M. Efecto analgésico de sevoflurano aplicado tópicamente sobre úlceras varicosas dolorosas en pacientes ambulatorios. Rev Seher. 2013;1:16-9.

21. Imbernon-Moya A, Ortiz-de Frutos FJ, Sanjuan-Alvarez M, Portero-Sanchez I, Merinero-Palomares R, Alcazar V. Pain and analgesic drugs in chronic venous ulcers with topical sevoflurane use. J Vasc Surg. 2018;1-6. 